\title{
Investigación Clínica \\ Adenosina intracoronaria durante la angioplastía primaria para la preservación del flujo coronario
}

Nicolás Veas, Dante Lindefjeld, José Luis Winter, Martín Valdebenito, Manuel Méndez,

Osvaldo Pérez, Eduardo Guarda, Alejandro Martínez.

Laboratorio de Hemodinamia del Hospital Dr. Sótero del Río.

Departamento de Enfermedades Cardiovasculares de la Pontificia Universidad Católica de Chile.

Introducción: El uso de adenosina intracoronario durante la angioplastía coronaria ha sido controversial en los últimos años. El beneficio teórico en el flujo epicárdico y microcirculatorio (MC) no se ha demostrado categóricamente en estudios clínicos.

Objetivo: Evaluar el efecto de protección de la MC y del flujo epicárdico al utilizar adenosina intracoronaria durante la AP.

Métodos: Estudio clínico randomizado multicéntrico, caso-control con análisis post hoc ciego, en pacientes portadores de SCA con SDST. Un total de 122 pacientes aleatorizados 1:1, se consideró caso $(\mathrm{A}(+))$ aquel que se administró adenosina en dosis de 120 microgramos intracoronario en bolo y luego infusión periférica de $6 \mathrm{mg}$ en $33 \mathrm{ml}$ de suero fisiológico a pasar en 2-3 minutos Se evaluaron criterios clínicos, angiográficos y electrocardiográficos de reperfusión epicárdica y microvascular. Observadores ciegos evaluaron el conteo de cuadros TIMI (cTFC) y "blush" miocárdico (BM). Se compararon las características clínicas, angiográficas basales y los resultados angiográficos finales entre ambos grupos, usando t-Student, prueba de Mann-Whitney, Chi cuadrado y test exacto de Fisher según correspondiera. En todos los pacientes se evaluó la resolución del SDST con el score de ST. Además, se evaluó las posibles complicaciones por uso de adenosina intracoronaria.

Resultados: Entre 2012-2014 se reclutaron 122 pacientes. Al comparar las características basales entre el grupo $\mathrm{A}(+)$ vs los $\mathrm{A}(-)$ no hubo diferencias significati- vas en la edad (59+/-10 años para A(+) vs 58+/-10 años para $\mathrm{A}(-), \mathrm{p}: 0,97)$, ni en las comorbilidades. Al comparar las características angiográficas basales, no se encontró diferencias en los vasos culpables (ADA 44\% en $\mathrm{A}(+)$ vs $43 \%$ en $\mathrm{A}(-)$, p:0.57), en las cargas trombóticas (Alta carga: $69 \%$ para $\mathrm{A}(+)$ vs $74 \%$ para $\mathrm{A}(-), \mathrm{p}: 0.53)$, en el flujo TIMI pre (TIMI 0-1 86\% para ambos grupos, p:0,69), cTFC pre $(87+/-23$ cuadros en $\mathrm{A}(+)$ vs $88+/$ 25 cuadros en $A(-)$, p:0.99), Killip de ingreso (Killip $\mathrm{I}, 86 \%$ para $\mathrm{A}(+)$ vs $76 \%$ para $\mathrm{A}(-), \mathrm{p}: 0,11)$ y fracción de eyección $(51+/-8 \%$ para $\mathrm{A}(+)$ vs $48+/-9 \%$ para $(-)$, $\mathrm{p}: 0,61)$. Al evaluar los resultados angiográficos finales encontramos diferencias significativas en el flujo TIMI (TIMI $396 \%$ para grupo $\mathrm{A}(+)$ vs $74 \%$ para grupo $\mathrm{A}(-)$, $\mathrm{p}: 0,002)$. No encontramos diferencias significativas en el BM (Blush $373 \%$ para ambos grupos, p:0.74), el cTFC final (24+/-11 cuadros en $\mathrm{A}(+)$ vs $26+/-12$ cuadros en A(-), p:0,85). Si consideramos cTFC <23cuadros como éxito angiográficos, tampoco encontramos diferencias significativas $(56 \%$ para $\mathrm{A}(+)$ vs $53 \%$ para $\mathrm{A}(-), \mathrm{p}: 0,45)$. Por último tampoco hubo diferencias significativas con la resolución del segmento ST (44\% para $\mathrm{A}(+)$ vs $58 \%$ para $\mathrm{A}(-)$, p:0,126).

Conclusión: De acuerdo a los resultados obtenidos podemos inferir que la adenosina intracoronaria cumple un rol en la conservación óptima del flujo epicárdico coronario, pero sin influir en la microcirculación. Mayores estudios se requieren para determinar si se traduce en algún beneficio clínico.

\section{Correspondencia:}

Dr. Nicolás Veas Paredes

Laboratorio de Hemodinamia Hospital Sótero del Rio.

Av Concha y Toro 3459, 4to piso. Laboratorio de Hemodinamia.

Santiago, Chile. CP 8330024.

nicoveas@gmail.com 


\section{Intracoronary Adenosine during Primary Angioplasty and Coronary Flow preservation}

Background: The effect of Intracoronary adenosine for coronary flow preservation during primary PTCA is debatable. Clinical studies have not established a benefit of adenosine administration upon epicardic or microcirculatory flows.

Aim: to evaluate micro circulatory flow preservation after administration of intracoronary adenosine during primary PTCA.

Method: From 2012 to 2014, 122 patients with ST elevation myocardial infarction randomized to either adenosine of control (2:1) were included in a controlled clinical trial. Adenosine was administered in a 120 $\mathrm{mg}$ bolus followed by $6 \mathrm{mg}$ solution during 2 to $3 \mathrm{~min}$. Epicardic and micro vascular flows were evaluated through clinical, angiographic, electrocardiographic and reperfusion variables. TIMI (cTFC) and myocardial "blush" were measured by blind observers.

Results: Basal characteristics, namely age and co-morbidities were similar between groups. Also, the distribution of coronary vessels involved in MI was similar with a preponderance of the LAD artery. There was an high proportion of patients with an elevated thrombus load (Adenosine 69\%, controls 74\%) ; TIMI flow $0-1$ was $86 \%$ in both groups and TIMI cTFC was not different (adenosine: $87 \pm 23$, controls $88 \pm 25$ ). Over $75 \%$ of patients were Killip I, and the ejection fraction was slightly decreased (adenosine $51 \pm 8 \%$, controls $48 \pm 9 \%$, NS). In contrast, TIMI flow was significantly greater for adenosine (TIMI $396 \%$ for adenosine and $74 \%$ for controls, $\mathrm{p}=0.002$ ). No difference was observed in myocardial blush (B $373 \%$ in both groups) nor cTFC ( $24 \pm 11$ vs. $26 \pm 12$, respectively). Finally, regression of ST elevation was similar in both groups.

Conclusion: Intracoronary adenosine during PTCA in ST elevation MI was associated to a better epicardial but not microvascular flow. Further study is needed to evaluate the eventual clinical benefit of these effects. Keywords: adenosine, intracoronary; ST elevation myocardial infarction; primary angioplasty; coronary flow. 
Introducción: La microembolización y deterioro de la función microvascular son fenómenos frecuentes en pacientes con infarto agudo al miocardio sometidos a angioplastía coronaria primaria (AP), y esto se asocia a mayor morbi-mortalidad, pese al alto grado de reperfusión en los vasos epicárdicos logrado con la AP. La embolización distal de material trombótico condiciona una menor reperfusión miocárdica, que se asocia a desenlaces negativos a mediano y largo plazo ${ }^{1,2}$.

El uso de coadyuvantes tales como inhibidores IIb-IIIa y antitrombínicos ha demostrado mejorar los eventos clínicos y los dependientes de la microcirculación (MC). En nuestra práctica clínica esto no puede ser llevado a cabo de forma rutinaria por temas de costos en salud pública. La adenosina endógena genera un mejor flujo coronario, por medio de la vasodilatación, hiperemia y disminución de la injuria por reperfusión, pero parece ser insuficiente para demostrar beneficio agudo en la protección microvascular. Además, el uso de adenosina intracoronaria ha sido bastante debatido durante los últimos años. Desmet et al. no demostraron beneficio clínico ni microvascular, como tampoco disminución inmediata del tamaño del infarto como tampoco 4 meses post intervención, medido por resonacia magnética cardíaca ${ }^{7}$. Un estudio reciente sí ha demostrado beneficio de adenosina para mejorar la microcirculación en comparación al uso de nitroprusiato o placebo, sin presentar complicaciones significativas (estudio REOPEN $^{8}$ ).

El objetivo del presente trabajo es evaluar el beneficio sobre el flujo coronario epicárdico y sobre la MC con el uso de adenosina intracoronaria durante la AP.

\section{Métodos}

\section{Hipótesis:}

a) Existen diferencias significativas en la $\mathrm{MC}$ con el uso de Adenosina intracoronaria posterior a la AP por IAM c/ SDST .

b) La embolización distal y el compromiso del flujo microvascular en sujetos con IAM con SDST sometidos a angioplastía primaria será menor en pacientes en quienes se utiliza Adenosina intracoronaria en comparación con los sujetos controles.

Objetivo general; Evaluar el efecto de protección de la microcirculación durante la AP con la administración de Adenosina intracoronaria en comparación con sujetos controles.

Objetivos específicos: Comparar entre ambos grupos los resultados de la microcirculación evaluado a través de conteo de cuadros (TIMI frame count (cTFC)), "blush" miocárdico (BM) y "score" de ST (ST-S).

\section{Objetivos Secundarios:}

- Medición de Flujo TIMI incial y post AP entre ambos grupos.

- Comparar el porcentaje de casos con cTFC $\leq 23$ post AP entre ambos grupos (10)

- Evaluar el grado de carga trombótica en cada grupo, con el "Thrombus TIMI score".

- Determinar el porcentaje de casos que obtienen regresión de $\geq 50 \%$ de ST-S post AP entre ambos grupos.

- Evaluar la seguridad del uso de adenosina intracoronaria en AP.

\section{Diseño y protocolo:}

Se llevó a cabo un estudio clínico multicéntrico (Hospital Clínico UC, Hospital Dr. Sótero del Río, Clínica UC San Carlos de Apoquindo), randomizado ciego y prospectivo con análisis post hoc ciego. En pacientes sometidos a AP con el diagnóstico de IAM con SDST con menos de doce horas de evolución se randomizaron en proporción 1:1 (en forma centralizada) a Adenosina 120 microgramos intracoronario en bolo y luego infusión periférica de $2 \mathrm{mg}$ en $33 \mathrm{ml}$ de suero fisiológico administrada en 2-3 min. vs. solución salina como control. Posterior al procedimiento, especialistas analizaron en forma retrospectiva y ciega las películas y electrocardiogramas evaluando indicadores de reperfusión: Flujo TIMI, cTFC y BM, ST-S.

Criterios de inclusión: Pacientes con IAM con SDST con $<12$ horas de evolución: hombres o mujeres con edad entre y 18 y 75 años con indicación de AP: Aceptación de consentimiento informado (o consentimiento de familiares directos): AP efectuada en vaso único, con diámetro $>2 \mathrm{~mm}$.

Criterios de exclusión: shock cardiogénico, trombolisis dentro de las últimas 24 horas, hemorragia intracraneal reciente, hemorragia digestiva reciente o síndrome ulceroso activo, trombosis intrastent, IAM secundario a oclusión de puentes aorto-coronarios o injertos mamarios, riesgo de bradicardia extrema, limitación crónica severa del flujo aéreo.

Angioplastía primaria: Se consideró AP al procedimiento realizado en pacientes con angina $>30$ minutos y presencia de SDST $>1 \mathrm{~mm}$, en al menos dos derivaciones contiguas. Los pacientes con IAM con SDST, previamente y de acuerdo a las normas vigentes, recibieron aspirina, betabloqueadores, nitroglicerina, heparina o analgésicos 
según correspondía en el servicio de urgencia. Durante la intervención, se mantuvo un tiempo de coagulación (ACT) $>300$ segundos, y se administró $600 \mathrm{mg}$ de clopidogrel o 180 mg de Ticagrelor según indicación del médico tratante. El uso de inhibidores IIb/IIIa, nitroglicerina, predilatación o postdilatación con balón fue a discreción del operador. Como pre requisito, para administrar la dosis de Adenosina intracoronaria y periférica se requirió tener la arteria coronaria con flujo a distal.

Cálculo muestral: En base a nuestro desenlace primario cTFC de $25 \pm 7$ en AP con uso de clopidogrel, asumiendo una reducción de al menos $20 \%$ en el cTFC al usar adenosina, se necesitan 45 pacientes por grupo, y al considerar una pérdida de $10 \%$ por diferentes motivos en ambos grupos se planificó incluir 55 pacientes por grupo.

El análisis estadístico se basó en: Descripción de las características basales de los pacientes (edad, sexo, comorbilidades, localización del infarto, horas de evolución, flujo TIMI inicial), Análisis del flujo TIMI inicial y post AP entre ambos grupos con la "prueba de $\mathrm{X}^{2}$ para muestras independientes" para variable de agrupación con $\mathrm{K} \geq 3$ grupos: Comparación del cTFC entre los resultados basales y post AP entre los grupos con el "test t de student para muestras independientes" o la "prueba U Mann - Whitney", Análisis del grado de BM basal y post AP entre los grupos mediante la "prueba de $\mathrm{X}^{2}$ para muestras independientes" para variable de agrupación con $\mathrm{K} \geq 3$ grupos y Kruskal Wallis, según corresponda, Evaluación de las diferencias en el grado de regresión del ST-S en cada uno de los grupos con el "test t de student para muestras independientes" ó la "prueba U Mann - Whitney", Comparación entre ambos grupos del porcentaje de casos que obtuvieron cTFC $\leq 23$ post angioplastía con la "prueba de $\mathrm{X}^{2}$ para muestras independientes" o el "test exacto de Fisher", Determinación del porcentaje de casos que obtienen regresión de $\geq$ $50 \%$ de ST post AP con la "prueba de $\mathrm{X}^{2}$ para muestras independientes" ó el "test exacto de Fisher".

Para los cálculos correspondientes se utilizó el paquete SPSS 15.0 y Excel (MS).

\section{Resultados}

Entre Junio del 2012 y Septiembre del 2014 se reclutaron 122 pacientes que cumplieron los criterios para ser incluidos, y aceptaron participar en el estudio. Los grupos quedaron conformados con 52 pacientes que recibieron Adenosina intracoronaria $(\mathrm{A}(+))$ y 70 pacientes que no la recibieron $(\mathrm{A}(-))$.
Características clínicas basales: Al comparar las caracterísiticas basales entre los 2 grupos no encontramos diferencias significativas en la edad, el porcentaje de hombres, la presencia de HTA, diabetes tipo 2, infartos previos, ni en la prevalencia de tabaquismo Solo la dislipidemia fue significativamente más frecuente en el Grupo A(+), (Tabla 1).

\begin{tabular}{|l|r|r|r|}
\hline \multicolumn{3}{|c|}{ Tabla 1: Características basales de los pacientes } \\
\hline & $\begin{array}{r}\text { Adenosina } \\
(+)(n=52)\end{array}$ & $\begin{array}{r}\text { Adenosina } \\
(-)(n=70)\end{array}$ & $p$ \\
\hline Sexo Masculino n(\%) & $37(71 \%)$ & $52(74 \%)$ & 0,43 \\
\hline Edad (años) & $58.9 \pm 9,9$ & $58,8 \pm 10,2$ & 0,98 \\
\hline Diabetes n(\%) & $20(38.5 \%)$ & $19(27.1 \%)$ & 0,13 \\
\hline Dislipidemia n(\%) & $27(51.9 \%)$ & $16(22.9 \%)$ & 0,001 \\
\hline Hipertensión n(\%) & $34(65.4 \%)$ & $41(58.6 \%)$ & 0,28 \\
\hline Tabaquismo n(\%) & $33(64 \%)$ & $43(61 \%)$ & 0,49 \\
\hline IAM previo n(\%) & $3(5.8 \%)$ & $2(2,9 \%)$ & 0,36 \\
\hline
\end{tabular}

Las principales características del IAM fueron comparables en ambos grupos, destacando tiempos de isquemia promedios entre 4.4 y 5.7 horas, FE de VI levemente disminuídas, y un predominio de pacientes en Killip 1. Respecto del vaso culpable, se trató mayoritariamente de la arteria descendente anterior (ADA) entre un cuarto y un tercio de los pacientes (Tabla 2).

\begin{tabular}{|c|c|c|c|}
\hline \multicolumn{4}{|c|}{$\begin{array}{l}\text { Tabla 2. Características del Infarto y vaso } \\
\text { culpable en ambos grupos }\end{array}$} \\
\hline & $\begin{array}{l}\text { Adenosina } \\
(+)(n=52)\end{array}$ & $\begin{array}{l}\text { Adenosina } \\
(-)(n=70)\end{array}$ & $p$ \\
\hline T. Isquemia (horas) & $5,7 \pm 3,3$ & $4,4 \pm 2,6$ & 0,05 \\
\hline Tiempo carga (min) & $45,2 \pm 15,4$ & $47,3 \pm 14,5$ & 0,34 \\
\hline FEVI (\%) & $51,1 \pm 8,1$ & $48,4 \pm 8,9$ & 0,61 \\
\hline Killip n(\%) & & & 0,28 \\
\hline 1 & $45(86,5 \%)$ & $53(76 \%)$ & \\
\hline 2 & $7(13,5 \%)$ & $26(23 \%)$ & \\
\hline 3 & $0(0 \%)$ & $1(1,4 \%)$ & \\
\hline 4 & $0(0 \%)$ & $0(0 \%)$ & \\
\hline Vaso Culpable n(\%) & & & 0,56 \\
\hline ADA & $23(44 \%)$ & $30(43 \%)$ & \\
\hline $\mathrm{Cx}$ & $13(25 \%)$ & $13(18 \%)$ & \\
\hline$C D$ & $16(31 \%)$ & 27 (39\%) & \\
\hline
\end{tabular}

$A D A$ : arteria descendente anterior; $C x$ : arteria circumfleja; $C D$ : arteria coronaria derecha. 
Respecto del flujo coronario, más del $80 \%$ de los pacientes tenían flujo TIMI 0 o 1 y el cTFC fue cercano a 90 en ambos grupos. La carga trombótica fue 4 a 5 en la mayoría de los pacientes. (Tabla 3 ).

\begin{tabular}{|l|r|r|r|}
\hline \multicolumn{4}{|c|}{$\begin{array}{c}\text { Tabla 3. Características basales del flujo } \\
\text { coronario y carga trombótica }\end{array}$} \\
\hline & $\begin{array}{r}\text { Adenosina } \\
(+)(n=52)\end{array}$ & $\begin{array}{r}\text { Adenosina } \\
(-)(n=70)\end{array}$ & $p$ \\
\hline Flujo TIMlpre (\%) & & & 0,23 \\
\hline 0 & $33(64 \%)$ & $52(74 \%)$ & \\
\hline 1 & $12(23 \%)$ & $8(11 \%)$ & \\
\hline 2 & $6(11 \%)$ & $6(9 \%)$ & \\
\hline 3 & $1(1,9 \%)$ & $4(5,7 \%)$ & \\
\hline cTFC pre & $87,3 \pm 22,7$ & $88,3 \pm 24,9$ & 0,99 \\
\hline Carga Trombótica & & & 0,57 \\
\hline $4-5(\%)$ & & \\
\hline
\end{tabular}

cTFC: conteo de cuadros TIMI

Procedimiento realizado: No encontramos diferencias significativas entre ambos grupos respecto de la aspiración de trombos (aproximadamente $45 \%$ ), predilatación (aproximadamente 64\%), número de Stents utilizados (aproximadamente 1.33 en promedio) y postdilatación (aproximadamente 34\%). El fenómeno de No reflow fue muy infrecuente y no significativamente diferente en ambos grupos.

Evaluación de la microcirculación posterior al procedimiento: $\mathrm{Al}$ evaluar los resultados angiográficos finales encontramos diferencias significativas en el flujo TIMI (TIMI 3 96\% para grupo $\mathrm{A}(+)$ vs $74 \%$ para grupo $\mathrm{A}(-)$, p:0,002) (Tabla 4). No encontramos diferencias significativas en el BM (3.7\% para ambos grupos), ni en el cTFC final (apropximadamente 25 cuadros). Si consideramos $<23$ cuadros como éxito angiográficos, tampoco encontramos diferencias significativas en relación al uso de adenosina (Figura 1). Por último, tampoco encontramos diferencias significativas en la resolución del segmento ST (44\% para $\mathrm{A}(+)$ vs $58 \%$ para $\mathrm{A}(-), \mathrm{p}: 0,126)$.

\section{Discusión:}

Los resultados de este estudio mostraron que la adenosina intracoronaria mejora el flujo coronario epicárdico obteniéndose flujos TIMI 3 en, aproximadamente, $30 \%$ más en los sujetos tratados en comparación con los controles. Sin embargo, medido por diferentes variables, no se demostró

\begin{tabular}{|l|r|r|r|}
\hline \multicolumn{4}{|c|}{ Tabla 4. Efecto sobre el flujo epicárdico post PTCA } \\
\hline & $\begin{array}{c}\text { Adenosina } \\
(+)(n=52)\end{array}$ & $\begin{array}{r}\text { Adenosina } \\
(-)(n=70)\end{array}$ & $p$ \\
\hline “No Reflow" n(\%) & $0(0 \%)$ & $4(13 \%)$ & 0,07 \\
\hline cTFC post n & $24,3 \pm 10,9$ & $25,9 \pm 11,6$ & 0,85 \\
\hline "Blush" Miocárdico & & & 0,74 \\
\hline 1 & $2(3,8 \%)$ & $5(7,1 \%)$ & \\
\hline 2 & $12(23,1 \%)$ & $14(20 \%)$ & \\
\hline 3 & $38(73,1 \%)$ & $51(72,9 \%)$ & \\
\hline Flujo TIMl post & & & 0,002 \\
\hline 2 & $2(3,8 \%)$ & $15(21 \%)$ & \\
\hline 3 & $50(96,2 \%)$ & $52(74)$ & \\
\hline
\end{tabular}

CTFC: conteo de cuadros TIMI

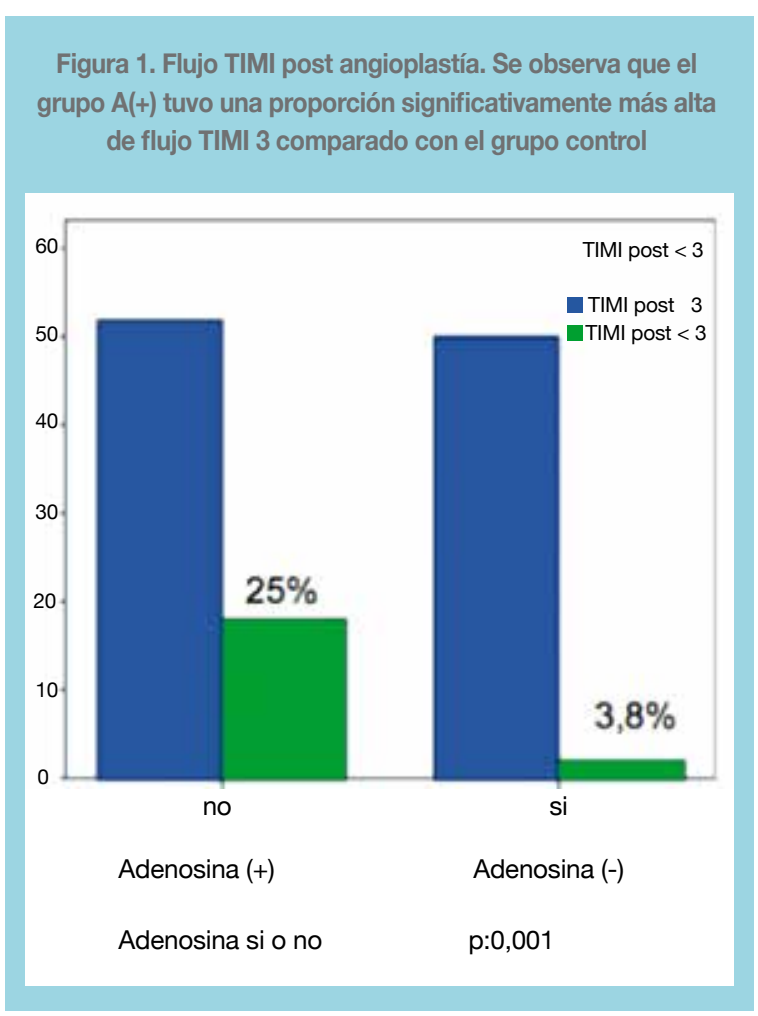

una mejoría de la circulación microvascular, como tampoco del tiempo de resolución del supradesnivel de ST.

El uso de adenosina intracoronario ha sido tema de debate a nivel mundial. Quedó en descrédito por el estudio publicado por Desmet et al., sin embargo, ha generado algunas críticas por las dosis y el mecanismo de infusión de la adenosina ${ }^{7}$. Por otro lado, el estudio REOPEN ${ }^{8}$ demostró beneficio sobre la microcirculación disminuyendo el cTFC final de forma significativa en comparación con nitropru- 

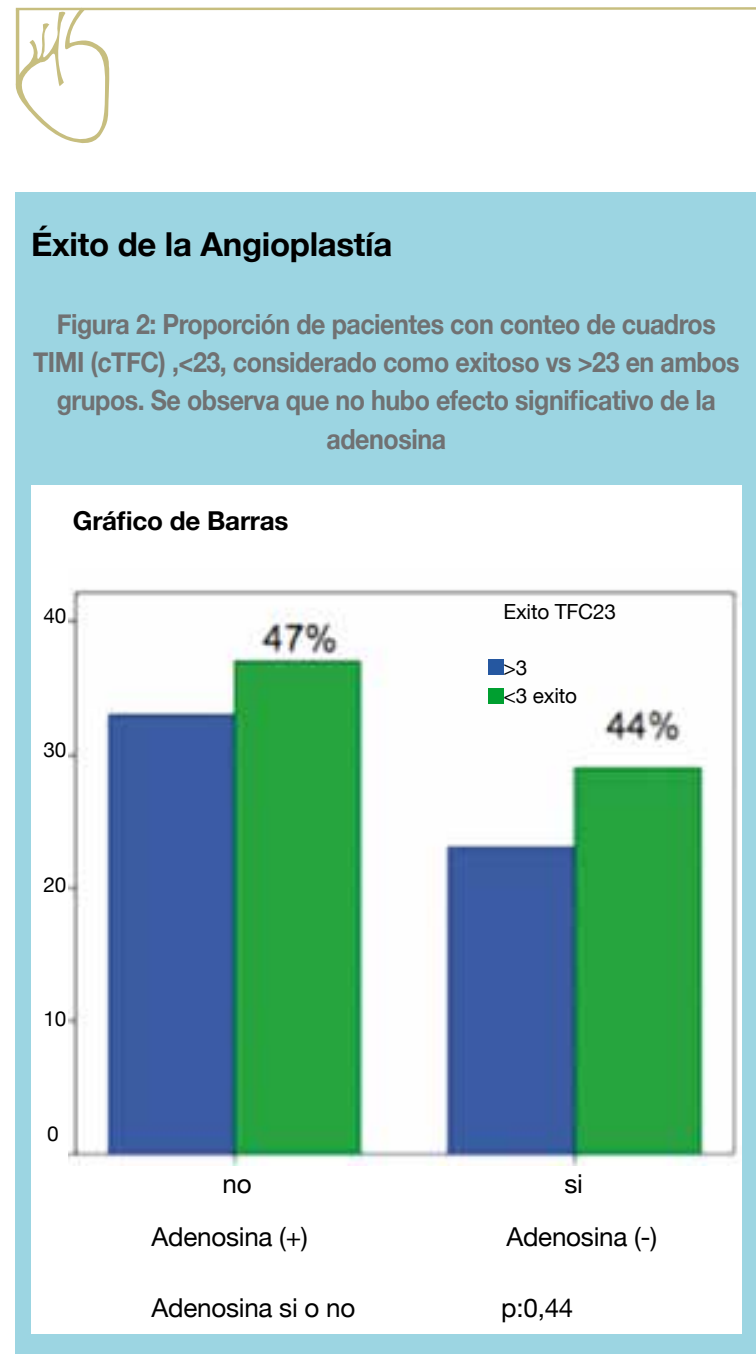

siato y placebo. Los autores enfatizan que para tener resultados positivos se debe administrar la adenosina una vez que se realice tromboaspiración y que la arteria esté permeable a distal. En nuestro estudio no encontramos diferencias significativas en lo que respecta a la microcirculación, pero si encontramos diferencias en el flujo epicárdico, siendo mejor para el grupo que se utiliza adenosina.
El beneficio a largo plazo ha sido demostrado en diversos estudios $^{1}$. En teoría, la adenosina mejoraría el flujo coronario mediante vasodilatación, hiperemia y disminución de la injuria por reperfusión, mejorando los flujos TIMI finales. Creemos que para obtener resultados como los nuestros, es importante tener la arteria con flujo a distal. En nuestro estudio aumentamos la dosis de infusión periférica en comparación con el estudio REOPEN, lo que también podría mejorar los resultados sobre el flujo epicárdico.

En nuestro medio, dado las condiciones económicas y la dificultad de utilizar coadyuvantes, es necesario crear nuevas herramientas para mejorar los desenlaces en angioplastía primaria en contexto de IAM con SDST. Los nuevos antiagregantes como ticagrelor o prasugrel, también han demostrado beneficio clínico, pero presentan la misma dificultad económica de mantener a los pacientes con estos medicamentos, generando menor adherencia a la terapia. Es por ello que nos parece que utilizar Adenosina en las dosis y vía de administración que nosotros planteamos, ayudaría en mejorar los flujos coronarios finales, con menores costos en salud.

\section{Conclusiones:}

De acuerdo a los resultados obtenidos podemos inferir que la adenosina intracoronaria cumple un rol en la conservación óptima del flujo épicardico coronario, pero sin influir en la microcirculación medido por cTFC, BM y Score ST. Mayores estudios se requieren para determinar si estos efectos se traducen en algún beneficio clínico. Nos parece que nuestro procedimiento se ajusta más a las posibilidad de la realidad nacional que no permite emplear los métodos utilizados en las principales publicaciones sobre el tema. 


\section{Referencias:}

1. CANNON C. Importance of TIMI 3 flow. Circulation. 2001; 104: $624-6$.

2. CURA FA, L'ALLIER PL, KAPADIA SR, HOUGHTALING PL, DIPAOLA LM, ELLIS SG, et al. Predictors and prognosis of suboptimal coronary blood flow after primary coronary angioplasty in patients with acute myocardial infarction. Am J Cardiol. 2001; 88: 124 - 8.

3. HAMADA $S$, NISHIUE T, NAKAMURA $S$, SUGIURA T, KAMIHATA H, MIYOSHI H, et al. TIMI frame count immediately after primary coronary angioplasty as a predictor of functional recovery in patients with TIMI 3 reperfused acute myocardial infarction. J Am Coll Cardiol. 2001; 38: 666 - 71.

4. EECKHOUT E, KERN MJ. The coronary no-reflow: a review of mechanisms and therapies. Eur Heart J. 2001; 22: 729 - 39.

5. KUNADIAN B, DUNNING J, VIJAYALAKSHMI K, THORNLEY AR, DE BELDER MA. Meta-analysis of randomized trials comparing anti-embolic devices with standard PCI for improving myocardial reperfusion in patients with acute myocardial infarction. CatheterCardiovasc Interv. 2007; 69: 488 - 96.

6. STONE GW, WEBBJ, COX DA, BRODIE BR, QUERESHI $\mathrm{M}, \mathrm{KALYNYCH} A$, et al. Distal microcirculatory protection during percutaneous coronary intervention in acute ST-segment elevation myocardial infarction: a randomized controlled trial. JAMA 2005; 293: 1063 - 72 .

7. DESMET W, BOGAERT J, DUBOIS C, SINNAEVE P, ADRIAENSSENS T, PAPPAS C, et al. High-dose intracoronary adenosine formyocardial salvage in patients with acute ST-segment elevation myocardial infarctionEur Heart J. 2011 Apr;32(7):867-77

8. NICCOLI G, RIGATTIERI S, DE VITA MR, VALGIMIGLI M, CORVO P, FABBIOCCHI F, et al. Open-Label, Randomized, Placebo-Controlled Evaluation of Intracoronary Adenosine o Nitroprusside After Thrombus Aspiration DuringcPrimary Percutaneous Coronary Intervention for the Prevention of Microvascular Obstruction in Acute Myocardial Infarction. J Am Coll Cardiol 2013; 6, 580-9.

9. GIBSON CM, CANNON CP, DALEY WL, DODGE JT JR, ALEXANDER B JR, MARBLE SJ, et al. TIMI frame count: a quantitative method of assessing coronary artery flow. Circulation. 1996; 93: $879-88$.

10. LINDEFJELD DS, GUARDA E, MÉNDEZ M, MARTÍNEZ A, PÉREZ O, FAJURI A, et al. Microvascular coronary flow comparison in acute myocardial infarction angioplasty treated with a mesh covered stent (MGUARD stent) versus bare metal stent: MICAMI-MGUARD.. Cardiovasc Revasc Med. 2013; 14: 4-8.

11. GOROG DA, FOALE RA, MALIK I. Distal myocardial protection during percutaneous coronary intervention: when and where? J Am Coll cardiol. 2005; 46: 1434 - 45.

12. SVILAAS T, VLAAR PJ, VAN DER HORST I, DIERCKS GF, DE SMET BJ, VAN DEN HEUVEL AF, et al. thrombus aspiration during primary percutaneous coronary intervention. N Engl J Med 2008; 358: 557 - 67.

13. VAN 'T HOF AW, LIEM A, SURYAPRANATA H, HOORNTJE JC, DE BOER MJ, ZIJLSTRA F. Angiographic assessment of myocardial reperfusion in patients treated with primary angioplasty for acute myocardial infarction: myocardial blush grade. Circulation 1998; 97: 2302 - 6 .

14. CLAEYS MJ, BOSMANS J, VEENSTRA L, JORENS P, DE RAEDTH, VRINTS CJ. Determinants and prognostic implications of persistent ST-segment elevation after primary angioplasty for acute myocardial infarction: importance of microvascular reperfusion injury on clinical outcome. Circulation. 1999; 99: $1972-7$

15. BERAN G, LANG I, SCHREIBER W, DENK S, STEFENELLI T, SYEDA B, et al. Intracoronary thrombectomy with the $\mathrm{X}$-Sizer catheter system improves epicardial flow and accelerates ST-Segment resolution in patients with acute coronary syndrome. Circulation. 2002; 105: 2355 - 60. 